Divyani Gandhi and Shikha Agarwal*

\title{
Urea nitrate catalyzed synthesis of 2-arylbenzothiazoles using the grindstone technique
}

https://doi.org/10.1515/hc-2018-0133

Received August 8, 2018; accepted October 16, 2018; previously published online November 13, 2018

Abstract: An efficient, green and environmentally friendly grindstone method for the synthesis of 2-arylbenzothiazole derivatives in the presence of urea nitrate as the catalyst under solvent-free conditions was developed. All products were characterized by elemental analysis and spectral methods.

Keywords: 2-aminobenzenethiol; 2-arylbenzothiazole; green synthesis; grindstone technique; urea nitrate.

\section{Introduction}

Benzothiazole is a versatile pharmacophore, possessing a wide spectrum of biological activities [1-13]. Accordingly, efficient approaches to the synthesis of benzothiazole derivatives, including green chemistry, are of interest. The important characteristics of green chemistry are the recovery of the catalyst after the catalytic reaction and minimization of waste. In this report a green synthesis of 2-arylbenzothiazoles using fine grinding of substituted 2-aminobenzenethiol and substituted aldehyde using a mortar and pestle under solvent-free conditions in the presence of urea nitrate catalyst is described. Urea nitrate is easily prepared in the laboratory and has been used previously to catalyze various organic transformations including the imino Diels-Alder reaction [14], regioselective nitration [15, 16], aromatization of 1,4-dihydropyridines [17] and cleavage of oximes under microwave irradiation [18] among other transformations. CAUTION: Urea nitrate is unstable and even at room temperature $(r t)$ slowly releases nitric acid. As it is a powerful explosive [19]

\footnotetext{
*Corresponding author: Shikha Agarwal, Synthetic Organic Chemistry Laboratory, Department of Chemistry, Mohanlal Sukhadia University, Udaipur 313001, India, e-mail: gandhi.divyani07@gmail.com, shikha_urj@yahoo.com Divyani Gandhi: Synthetic Organic Chemistry Laboratory, Department of Chemistry, Mohanlal Sukhadia University, Udaipur 313001, India
}

it must be handled with care [20,21]. Urea nitrate can easily be neutralized by treatment with water.

\section{Results and discussion}

In continuation of our research, an attempt was made to synthesize some derivatives of 2-arylbenzothiazole derivatives from 2-aminobenzenethiol under environmental benign conditions using urea nitrate as a catalyst via the grindstone method. The compounds 3a-f were synthesized by performing organic transformations under solvent-free conditions. The reaction has previously been performed under different conditions (Table 1) using $\mathrm{ZnO}$ NPs [22], ZnO NPs (ball milling strategy) [23], tetrabutylammonium bromide (TBAB) [24], $\mathrm{TiO}_{2}$ [25], glycerol [26], tungstophosphoric acid impregnated zirconium phosphate [27], sulfonated porous carbon (SPC) [28], calcined eggshell [29] and liquid-assisted hand grinding [30], among other methods. Most of the synthetic approaches reported in the literature are characterized by high yields and short reaction times but the catalysts used are impractical and hazardous solvents are used along with tedious isolation procedures. By contrast, the synthesis of urea nitrate is simple, economical and the products $\mathbf{3 a - f}$ are obtained without using solvents and avoiding tedious isolation procedures. Bearing in mind the importance of urea nitrate as a catalyst, we made efforts to develop an environment friendly method using the grindstone technique. Our method involves grinding substituted 2-aminobenzenethiol and various aldehydes with a mortar and pestle using urea nitrate as a catalyst and the product is obtained in a few seconds, visualized by a dramatic color change. Excellent yields are obtained without a tedious purification process of column chromatography. The reaction is carried out at $\mathrm{rt}$ in the absence of any solvent with different substituted thiols $\mathbf{1}$ and aromatic aldehydes $\mathbf{2}$ in the presence of urea nitrate and produces compounds 3a-f (Scheme 1). Compounds 3d,e and $\mathbf{3 f}$ have been synthesized previously using different methods [31, 32] but the yields and reaction times have not been satisfactory. In this work, these compounds were resynthesized using 
Table 1 Comparative study of various catalysts.

\begin{tabular}{|c|c|c|c|c|}
\hline Entry & Catalyst & Reaction condition & Time & Yield (\%) \\
\hline 1 & Urea nitrate (15 mol\%)a & Grinding, solvent free, rt & $30 \mathrm{~s}$ & 98 \\
\hline 2 & $\mathrm{ZnO}$ NPs (5 mg) [22] & Solvent free or ethanol, rt & $2-8 \mathrm{~min}$ & 95 \\
\hline 3 & ZnO NPs [23] & Ball milling, $600 \mathrm{rpm}, \mathrm{rt}$ & $30 \mathrm{~min}$ & 87 \\
\hline 4 & Tetrabutylammonium bromide (TBAB) (15 mol\%) [24] & Reflux at $60^{\circ} \mathrm{C}$ & $30 \mathrm{~min}$ & 90 \\
\hline 5 & $\mathrm{TiO}_{2} \mathrm{P} 25(0.1) / \mathrm{H}_{2} \mathrm{O}_{2}[25]$ & Ambient-light conditions at $50^{\circ} \mathrm{C}$ & $5 \mathrm{~min}$ & 96 \\
\hline 6 & Glycerol $(2 \mathrm{~mL})[26]$ & Microwave irradiation at $100^{\circ} \mathrm{C}$ & $4 \mathrm{~min}$ & 92 \\
\hline 7 & Tungstophosphoric acid impregnated zirconium phosphate (5 mol\%) [27] & Microwave irradiation at $100^{\circ} \mathrm{C}$ & $15 \mathrm{~min}$ & 95 \\
\hline 8 & Sulfonated porous carbon (SPC) $(0.1 \mathrm{~g})[28]$ & Microwave irradiation in water & $6 \mathrm{~min}$ & 93 \\
\hline 9 & Calcined egg shell [29] & Grinding, solvent free, rt & $16 \mathrm{~min}$ & 92 \\
\hline
\end{tabular}

apresent work.<smiles>[R]c1cc([R2])c(S)c(N)c1</smiles>

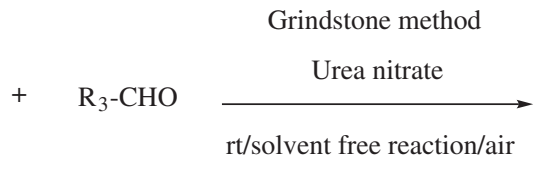

2<smiles>[R]c1cc([R2])c2sc([R3])nc2c1</smiles>

3a-f

$\begin{array}{cccl} & \mathbf{R}_{\mathbf{1}} & \mathbf{R}_{\mathbf{2}} & \mathbf{R}_{\mathbf{3}} \\ \text { 3a } & \mathrm{CH}_{3} & \mathrm{CH}_{3} & \text { 4-cyanophenyl } \\ \text { 3b } & \mathrm{CH}_{3} & \mathrm{CH}_{3} & \text { 3,4-dimethoxyphenyl } \\ \text { 3c } & \mathrm{CH}_{3} & \mathrm{CH}_{3} & \text { 2-furanyl } \\ \text { 3d } & \mathrm{H} & \mathrm{H} & \text { 3,4-dimethoxyphenyl } \\ \text { 3e } & \mathrm{H} & \mathrm{H} & \text { 4-ethoxyphenyl } \\ \text { 3f } & \mathrm{H} & \mathrm{H} & \text { 2-furanyl }\end{array}$

Scheme 1

urea nitrate as a catalyst via the grinding procedure. It was found that the rate of reaction increased continuously with the increase in the catalyst concentration up to $15 \mathrm{~mol} \%$, which was found to be the optimal concentration. The optimum reaction time (grinding) was $30 \mathrm{~s}$. The reaction was also attempted in the presence of $\mathrm{HNO}_{3}$ and urea alone under otherwise similar conditions but the product was obtained in a lower yield along with a longer reaction time. The structures of all synthesized compounds were confirmed by analysis of IR and NMR spectra.

\section{Conclusion}

An efficient and green procedure for the synthesis of 2-arylbenzothiazoles from substituted 2-aminobenzenethiol was developed. The work is highly advantageous because of easy workup, solvent-free conditions, high yields, no waste generation and the ease of product isolation and purification.

\section{Experimental}

All chemicals were commercially procured from Sigma-Aldrich and Hi-Media and used without further purification. An agate mortar and pestle was used for the synthesis. Melting points were determined in open capillary tubes and are uncorrected. The IR spectra were recorded in $\mathrm{KBr}$ pellets on a Bruker FT-IR spectrometer. The ${ }^{1} \mathrm{H}$ NMR (400 MHz) and ${ }^{13} \mathrm{C}$ NMR (100 MHz) spectra were scanned on a Bruker Avance II 400 spectrometer using tetramethylsilane (TMS) as an internal standard and $\mathrm{CDCl}_{3}$ as a solvent. The mass spectra were recorded on a Waters Xevo G2-S QT spectrometer. The purity of synthesized compounds was checked by thin layer chromatography (TLC) using silica gel G as an adsorbent and eluting with hexanes/ 
ethyl acetate; visualization was accomplished by UV irradiation or exposure to iodine. The starting material 2-amino-4,6-dimethyl-benzenethiol was synthesized as previously reported [33].

\section{Synthesis of urea nitrate catalyst}

Urea (3 g) in a 100-mL beaker was treated dropwise by stirring with $\mathrm{HNO}_{3}(10 \mathrm{~mL})$. A clear solution was formed upon heating. The solution was cooled to rt and the resultant bright shiny crystals of urea nitrate were filtered, dried and stored in air tight vials. The catalyst is unstable in the presence of water.

\section{General procedure for synthesis of 2-phenylbenzothia- zoles $3 a-f$}

An aromatic aldehyde (1 mol), $o$-aminothiophenol (1 mmol) and urea nitrate $(15 \mathrm{~mol} \%)$ were grounded together using a mortar and pestle at $\mathrm{rt}$ for few seconds giving rise to a dramatic color change as the reaction progressed. The resultant solid mass was treated with ice-cold water and the mixture was stirred for $30 \mathrm{~min}$ to remove urea. After filtration, the solid residue of $\mathbf{6 a - f}$ was crystallized from ethanol.

4-(5,7-Dimethyl-benzothiazol-2-yl)-benzonitrile (3a) Yield 96\%; dark yellow crystals; mp 212-215 ${ }^{\circ} \mathrm{C}$; IR: 3051 (C-H stretching, Ar), 2965 (C-H stretching, $\left.\mathrm{CH}_{3}\right), 2213$ (-CN, stretching) $1470 \mathrm{~cm}^{-1}$ (aromatic $\mathrm{C}=\mathrm{C}$ ); ${ }^{1} \mathrm{H}$ NMR: $\delta 2.19$ (s, 3H, $\mathrm{CH}_{3}$ ), 2.21 (s, 3H, $\mathrm{CH}_{3}$ ), 7.26-7.79 (m, 6H, Ar-H and BT-H); ${ }^{13}$ C NMR: $\delta$ 20.3, 21.6, 115.9, 117.0, 124.4, 127.1, 127.5, 129.9, 132.3, 132.9, 134.5, 136.4, 137.3, 139.0, 153.1, 166.2; MS (EI): $m / z 264.89$ $[\mathrm{M}+]$. Anal. Calcd for $\mathrm{C}_{16} \mathrm{H}_{12} \mathrm{~N}_{2} \mathrm{~S} ; \mathrm{C}, 72.70 ; \mathrm{H}, 4.58 ; \mathrm{N}, 10.60$. Found: C, 72.53; H, 4.52; N, 10.65 .

2-(3,4-Dimethoxy-phenyl)-5,7-dimethyl-benzothiazole (3b) Yield 95\%; red orange crystals; mp $174-177^{\circ} \mathrm{C}$; IR: $3110(\mathrm{C}-\mathrm{H}$ stretching, Ar), 2973 (C-H stretching, $\mathrm{CH}_{3}$ ), $1452 \mathrm{~cm}^{-1}$ (aromatic $\mathrm{C}=\mathrm{C}$ ); ${ }^{1} \mathrm{H}$ NMR: $\delta 2.26\left(\mathrm{~s}, 3 \mathrm{H}, \mathrm{CH}_{3}\right), 2.30\left(\mathrm{~s}, 3 \mathrm{H}, \mathrm{CH}_{3}\right), 3.68\left(\mathrm{~s}, 3 \mathrm{H}, \mathrm{OCH}_{3}\right), 3.82$ (s, 3H, $\mathrm{OCH}_{3}$ ), 6.95-7.87 (m, 5H, Ar-H and BT-H); ${ }^{13} \mathrm{C}$ NMR: $\delta 20.5,21.8$, 56.2, 57.4, 111.0, 112.2, 123.4, 128.8, 130.3, 131.5, 131.9, 138.5, 138.9, 148.3, 149.1, 152.6, 166.5; MS (EI): $m / z$ 299.47 [M+]. Anal. Calcd for $\mathrm{C}_{17} \mathrm{H}_{17} \mathrm{NO}_{2} \mathrm{~S} ; \mathrm{C}, 68.20 ; \mathrm{H}, 5.72 ; \mathrm{N}, 4.68$. Found: C, 65.30; H, 5.82; N, 4.71.

2-Furan-2-yl-5,7-dimethyl-benzothiazole (3c) Yield 98\%; dark purple crystals; mp $182-185^{\circ} \mathrm{C}$; I: 3085 (C-H stretching, Ar), 2943 (C-H stretching, $\mathrm{CH}_{3}$ ), $1473 \mathrm{~cm}^{-1}$ (aromatic $\left.\mathrm{C}=\mathrm{C}\right)$; ${ }^{1} \mathrm{H}$ NMR: $\delta 2.32\left(\mathrm{~s}, 3 \mathrm{H}, \mathrm{CH}_{3}\right.$ ), 2.38 (s, 3H, $\mathrm{CH}_{3}$ ), 6.63 (dd, 1H, J=3.4, $1.8 \mathrm{~Hz}$ ), 7.08 (d, 2H, BT-H), 7.17 (dd, $1 \mathrm{H}, J=3.4,0.8 \mathrm{~Hz}$ ), 7.87 (dd, $1 \mathrm{H}, J=1.8,0.8 \mathrm{~Hz}$ ); ${ }^{13} \mathrm{C}$ NMR: $\delta 20.9$, 21.4, 108.8, 112.3, 122.7, 129.7, 131.0, 137.0, 138.4, 144.1, 149.1, 151.1, 156.6; MS (EI): $m / z$ 229.74 [M+]. Anal. Calcd for $\mathrm{C}_{13} \mathrm{H}_{11} \mathrm{NOS}$; C, 68.09; H, 4.84; N, 6.11. Found: C, 68.11; H, 4.93; N, 6.23.

2-(3,4-Dimethoxy-phenyl)-benzothiazole (3d) Yield 98\%; dark yellow crystals; mp $136-139^{\circ} \mathrm{C}$; IR: 3120 (C-H stretching, Ar), 3053 (C-H stretching, $\mathrm{CH}_{3}$ ), $1465 \mathrm{~cm}^{-1}$ (aromatic $\mathrm{C}=\mathrm{C}$ ); ${ }^{1} \mathrm{H}$ NMR: $\delta 3.68$ (s, $\left.3 \mathrm{H}, \mathrm{OCH}_{3}\right), 3.84\left(\mathrm{~s}, 3 \mathrm{H}, \mathrm{OCH}_{3}\right), 6.94-7.46(\mathrm{~m}, 7 \mathrm{H}, \mathrm{Ar}-\mathrm{H}$ and $\mathrm{BT}-\mathrm{H}) ;{ }^{13} \mathrm{C}$ NMR: $\delta$ 56.1, 57.3, 111.6, 113.2, 122.4, 122.8, 124.9, 126.4, 128.4, 131.0, 134.1, 148.5, 149.1, 153.4, 163.5; MS (EI): $m / z$ 271.82 [M+]. Anal. Calcd for $\mathrm{C}_{15} \mathrm{H}_{13} \mathrm{NO}_{2} \mathrm{~S} ; \mathrm{C}, 66.40 ; \mathrm{H}, 4.83$; N, 5.16. Found: C, 66.57; H, 4.93; N, 5.26.
2-(4-Ethoxy-phenyl)-benzothiazole (3e) Yield 95\%; gray crystals; mp 117-120 ${ }^{\circ}$; IR: 3152 (C-H stretching, Ar), 3087 (C-H stretching, $\left.\mathrm{CH}_{3}\right), 2925\left(\mathrm{CH}_{2}\right.$, C-H stretching), $1460 \mathrm{~cm}^{-1}$ (aromatic $\left.\mathrm{C}=\mathrm{C}\right)$; ${ }^{1} \mathrm{H}$ NMR: $\delta 1.27\left(\mathrm{t}, 3 \mathrm{H}, \mathrm{CH}_{3}, J=7.0 \mathrm{~Hz}\right.$ ), 4.14 (q, 2H, $\mathrm{CH}_{2}, J=7.0 \mathrm{~Hz}$ ), 7.12-7.97 (m, 8H, Ar-H and BT-H); ${ }^{13} \mathrm{C}$ NMR: $\delta 14.7,63.6,114.5,114.7,122.7,124.1,125.2$, 126.9, 127.3, 128.1, 128.5, 135.4, 154.3, 161.0, 167.1; MS (EI): $m / z 255.68$ $[\mathrm{M}+]$. Anal. Calcd for $\mathrm{C}_{15} \mathrm{H}_{13}$ NOS: C, 70.56; H, 5.13; N, 5.49. Found: C, 70.68; H, 5.19; N, 5.55 .

2-Furan-2-yl-benzothiazole (3f) Yield 97\%; dark purple crystals; mp $105-108^{\circ} \mathrm{C}$; IR: 3080 (C-H stretching, Ar), $1458 \mathrm{~cm}^{-1}$ (aromatic $\mathrm{C}=\mathrm{C})$; ${ }^{1} \mathrm{H}$ NMR: $\delta 6.70(1 \mathrm{H}, \mathrm{dd}, J=3.4,1.8 \mathrm{~Hz}), 7.19(1 \mathrm{H}, \mathrm{dd}, J=3.4$, $0.9 \mathrm{~Hz}), 7.30-8.09$ (m, 4H, BT-H), $7.98(1 \mathrm{H}, \mathrm{dd}, J=1.8 \mathrm{~Hz}$ and $0.9 \mathrm{~Hz})$; ${ }^{13} \mathrm{C}$ NMR: $\delta 108.8,112.3,122.6,124.6,124.8,126.3,133.8,144.1,149.1$, 153.1, 156.6; MS (EI): $m / z$ 201.59 [M+]. Anal. Calcd for $\mathrm{C}_{11} \mathrm{H}_{7} \mathrm{NOS}: \mathrm{C}$, 65.65; H, 3.51; N, 6.96. Found: C, 65.76; H, 3.58; N, 6.93.

Acknowledgment: The authors are grateful to Department of Chemistry, MLSU for providing laboratory facilities. Analytical facilities by SAIF Chandigarh are duly acknowledged.

\section{References}

[1] Kumbhare, R. M.; Kosurkar, U. B.; Ramaiah, M. J.; Dadmal, T. L.; Pushpavalli, S. N. C. V. L.; Bhadra, M. P. Synthesis and biological evaluation of novel triazoles and isoxazoles linked 2-phenyl benzothiazole as potential anticancer agents. Bioorg. Med. Chem. Lett. 2012, 22, 5424-5427.

[2] Hiyoshi, H.; Goto, N.; Tsuchiya, M.; lida, K.; Nakajima, Y.; Hirata, N.; Kanda, Y.; Nagasawa, K.; Yanagisawa, J. 2-(4-hydroxy3-methoxyphenyl)-benzothiazole suppresses tumor progression and metastatic potential of breast cancer cells by inducing ubiquitin ligase CHIP. Sci. Rep. 2014, 4, 7095.

[3] Bandyopadhyay, P.; Sathe, M.; Ponmariappan, S.; Sharma, A.; Sharma, P.; Srivastava, A. K.; Kaushik, M. P. Exploration of in vitro time point quantitative evaluation of newly synthesized benzimidazole and benzothiazole derivatives as potential antibacterial agents. Bioorg. Med. Chem. Lett. 2011, 21, 7306-7309.

[4] Patel, N. B.; Shaikh, F. M. Synthesis and antimicrobial activity of new 4-thiazolidinone derivatives containing 2-amino-6-methoxybenzothiazole. Saudi Pharm. J. 2010, 18, 129-136.

[5] Cano, N. H.; Ballari, M. S.; Lopez, A. G.; Santiago, A. N. New synthesis and biological evaluation of benzothiazole derivates as antifungal agents. J. Agric. Food Chem. 2015, 63, 3681-3686.

[6] Pejchal, V.; Pejchalova, M.; Ruzickova, Z. Synthesis, structural characterization, antimicrobial and antifungal activity of substituted 6-fluorobenzo[d]thiazole amides. Med. Chem. Res. 2015, 24, 3660-3670.

[7] Zablotskaya, A.; Segal, I.; Geronikaki, A.; Eremkina, T.; Belyakov, S.; Petrova, M.; Shestakova, I.; Zvejniece, L.; Nikolajeva, V. Synthesis, physicochemical characterization, cytotoxicity, antimicrobial, anti-inflammatory and psychotropic activity of new $N$-[1,3-(benzo)thiazol-2-yl]- $\omega$-[3,4-dihydroisoquinolin-2(1H)-yl] alkanamides. Eur. J. Med. Chem. 2013, 70, 846-856. 
[8] Mahtab, R.; Srivastava, A.; Gupta, N.; Kushwaha, S. K.; Tripathi, A. Synthesis of novel 2-benzylbenzo[d] thiazole-6-sulfonamide derivatives as potential anti-inflammatory agent. J. Chem. Pharm. Sci. 2014, 7, 34-38.

[9] Siddiqui, N.; Rana, A.; Khan, S. A.; Haque, S. E.; Alam, M. S.; Ahsan, W.; Ahmed, S. Synthesis of 8-substituted-4-(2/4substituted phenyl)-2H-[1,3,5]triazino[2,1-b][1,3]benzothiazole2-thiones and their anticonvulsant, anti-nociceptive, and toxicity evaluation in mice. J. Enz. Inhib. Med. Chem. 2009, 24, 1344-1350.

[10] Yogeswari, P.; Sriram, D.; Mehta, S.; Nigam, D.; Kumar, M. M.; Murugesan, S. Stables, J. P. Anticonvulsant and neurotoxicity evaluation of some 6-substituted benzothiazolyl-2-thiosemicarbazones. Il Farmaco 2005, 60, 1-5.

[11] Ke, S.; Wei, Y.; Yang, Z.; Wang, K.; Liang, Y.; Shi, L. Novel cycloalkylthiophene-imine derivatives bearing benzothiazole scaffold: synthesis, characterization and antiviral activity evaluation. Bioorg. Med. Chem. Lett. 2013, 23, 5131-5134.

[12] Bhoi, M. N.; Borad, M. A.; Panchal, N. K.; Patel, H. D. 2-Aminobenzothiazole containing novel Schiff bases derivatives: Search for new antibacterial agents. Int. Lett. Chem. Phys. Astron. 2015, 53, 106-113.

[13] Bhoi, M. N.; Borad, M. A.; Parmar, H. B.; Patel, H. D. Synthesis and characterization of novel $\mathrm{N}$-(benzo[d]thiazol-2-yl)-2-(2(6-chloroquinolin-4-yl)hydrazinyl)acetamide derivatives containing quinoline linkage as potent antibacterial agents. Int. Lett. Chem. Phys. Astron. 2015, 53, 114-121.

[14] Anniyappan, M.; Nagarajan, R.; Perumal, P. T. Urea nitrate catalyzed imino Diels-Alder reactions: synthesis of cyclopentaquinolines, pyranoquinolines, and furoquinoline derivatives. Synth. Commun. 2002, 32, 99-103.

[15] Almog, J.; Klein, A.; Sokol, A.; Sasson, Y.; Sonenfeld, D.; Tamiri, T. Urea nitrate and nitrourea: powerful and regioselective aromatic nitration agents. Tetrahedron Lett. 2006, 47, 8651-8652.

[16] Nagarajan, R.; Muralidharan, D.; Perumal, P. T. A new and facile method for the synthesis of nitrocarbazoles by urea nitrate. Synth. Commun. 2004, 34, 1259-1264.

[17] Anniyappan, M.; Muralidharan, D.; Perumal, P. T. A novel application of the oxidizing properties of urea nitrate and peroxydisulfate-cobalt(II):aromatization of $\mathrm{NaD}(\mathrm{P}) \mathrm{H}$ model Hantzsch 1,4-dihydropyridines. Tetrahedron 2002, 58, 5069-5073.

[18] Perumal, P. T.; Anniyappan, M.; Muralidharan, D. Deprotection of oximes using urea nitrate under microwave irradiation. J. Chem. Sci. 2004, 116, 261-264.

[19] Medard, L. Explosive properties of urea nitrate, nitrourea, and guanidine nitrate. Memorial. Des. Poudres. 1951, 33, 113-123.

[20] The Health and Safety at Work Act 1974, Chapter 37, HMSO ISBN 0105437743.

[21] Oxley, J. C.; Smith, J. L.; Naik, S.; Moran, J. Decompositions of urea and guanidine nitrates. J. Energ. Mater. 2008, 27, 17-39.
[22] Banerjee, S.; Payra, S.; Saha, A.; Sereda, G. ZnO nanoparticles: a green efficient catalyst for the room temperature synthesis of biologically active 2-aryl-1,3-benzothiazole and 1,3-benzoxazole derivatives. Tetrahedron Lett. 2009, 55, 5515-5520.

[23] Sharma, H.; Singh, N.; Jang, D. O. A ball-milling strategy for the synthesis of benzothiazole, benzimidazole and benzoxazole derivatives under solvent-free conditions. Green Chem. 2014, 16, 4922-4930.

[24] Jain, R.; Sharma, K.; Kumar, D. A greener, facile and scalable synthesis of indole derivatives in water: reactions of indole2,3-diones with 1,2-difunctionalized benzene. Tetrahedron Lett. 2012, 53, 6236-6240.

[25] Bahramia, K.; Khodaeia, M. M.; Naalia, F. TiO ${ }_{2}$ nanoparticles catalysed synthesis of 2-arylbenzimidazoles and 2-arylbenzothiazoles using hydrogen peroxide under ambient light. J. Exp. Nanosci. 2016, 11, 148-160.

[26] Zhanga, X. Z.; Zhou, W. J.; Yanga, M.; Wangb, J. X.; Baic, L. Microwave-assisted synthesis of benzothiazole derivatives using glycerol as green solvent. J Chem. Res. 2012, 489-491.

[27] Aliyan, H.; Fazaeli, R.; Fazaeli, N.; Mssah, A. R.; Naghash, H. J.; Alizadeh, M.; Emami, G. Facile route for the synthesis of benzothiazoles and benzimidazoles in the presence of tungstophosphoric acid impregnated zirconium phosphate under solvent-free conditions. Heteroatom Chem. 2009, 20, 202-207.

[28] Shokrolahi, A.; Zali, A.; Mahdavi, M. Sulfonated porous carbon (SPC)-catalyzed synthesis of benzothiazole derivatives in water. Phosphorus Sulfur Silicon 2012, 187(4), 535-543.

[29] Borhade, A. V.; Uphade, B. K.; Gadhave, A. G. Calcinized eggshell: an environmentally benign green catalyst for synthesis of 2-arylbenzothiazole derivatives. Res. Chem. Intermed. 2016, 42, 6301-6311.

[30] Banerjee, M.; Chatterjee, A.; Kumar, V.; Bhutia, Z. T.; Khandare, D. G.; Majik, M. S.; Roy, B. G. A simple and efficient mechanochemical route for the synthesis of 2-aryl benzothiazoles and substituted benzimidazoles. RSC Adv. 2014, 4, 39606-39611.

[31] Bandyopadhyay, P.; Sathe, M.; Ponmariappan, S.; Sharma, A.; Sharma, P.; Srivastava, A. K.; Kaushik, M. P. Exploration of in vitro time point quantitative evaluation of newly synthesized benzimidazole and benzothiazole derivatives as potential antibacterial agents. Bioorg. Med. Chem. Lett. 2011, 21, 7306-7309.

[32] Kumar, P.; Meenakshi; Kumar, S.; Kumar, A.; Hussain, K.; Kumara, S. Solvent-free one pot synthesis of 2-aryl/heteroarylbenzothiazoles using hypervalent iodine (III) reagents. J. Heterocycl. Chem. 2012, 49, 1243-1249.

[33] Rathore, B. S.; Kumar, M.; Gupta, R. R. Synthesis of 7-chloro5-trifluoromethyl/7-fluoro/7-trifluoromethyl-4H-1,4-benzothiazines as antimicrobial agents. Biorg. Med. Chem. 2006, 14, 5678-5682. 